\title{
Comparative Study of Vasodilatation Induced by Rokumigan, Hachimijiogan, and Goshajinkigan in the Rat Aorta
}

Keywords: Rokumigan; Hachimijiogan; Goshajinkigan; Vasodilatation; Endothelium; Rat aorta

\begin{abstract}
Rokumigan, hachimijiogan, and goshajinkigan are traditional Japanese Kampo formulations with similar components and consequently similar clinical indications. These formulations are considered useful for the management of edema, water disturbances, dry skin, dry mouth, and abnormal urination. Rokumigan is composed of six types of crude herbal drugs, hachimijiogan comprises rokumigan and two crude drugs, and goshajinkigan includes hachimijiogan and two other crude drugs. In this study, we compared the vasodilatations induced by these formulations and investigated the underlying mechanisms using rat aortic ring strips. We found that 0.1 to $3.0 \mathrm{mg} /$ $\mathrm{mL}$ of these formulations counteracted the effects norepinephrine $(5$ $\mu \mathrm{M})$-induced vasoconstriction in a concentration dependent manner. The vasodilatation induced by 0.03 to $1.0 \mathrm{mg} / \mathrm{mL}$ of the formulations was similar, although rokumigan induced the greatest vasodilatation at $3.0 \mathrm{mg} / \mathrm{mL}$. Pretreatment with L-NAME $(100 \mu \mathrm{M})$ and endothelium removal significantly attenuated the vasodilatation induced by the three formulations, whereas indomethacin $(10 \mu \mathrm{M})$ showed no effects. Furthermore, pretreatments with propranolol $(3 \mu \mathrm{M})$ and nicardipine (2 $\mu \mathrm{M})$ markedly decreased this vasodilatation, which was also observed in $\mathrm{Ca}^{2+}-$ free solution. These results indicate that rokumigan, hachimijiogan, and goshajinkigan induce marked vasodilatation via nitric oxide production from the endothelium and via $\beta$-adrenoceptor stimulation and blockade of L-type $\mathrm{Ca}^{2+}$ channels on the vascular smooth muscle.
\end{abstract}

\section{Introduction}

Rokumigan, hachimijiogan and goshajinkigan, which are traditional Japanese herbal (Kampo) formulations, have been used for the management of conditions such as hypertension, chronic nephritis, low back pain, and prostatic hypertrophy [1]. The crude drug components in these three formulations are similar, although but the number of crude drugs varies. Rokumigan comprises six crude drugs (Rahmanniae Radix, Corni Fuructus, Dioscorea Rhizoma, Alismatis Rhizoma, Poria cocos, and Moutan Cortex); hachimijiogan comprises the six drugs of rokumigan and two additional crude drugs (Cinnamomi Cortex and Processi Aconiti Tuber); and goshajinkigan comprises the eight crude drugs of hachimijiogan and two additional drugs (Achyranthis Radix and Plantaginis Semen).

In the field of oriental medicine, rokumigan, hachimijiogan, and goshajinkigan are considered to regulate "Jin" (namely kidney) functions. The "Jin" has been considered to regulate the water balance in the body, urinary tract and hearing systems, bone and bone marrow, sexual energy, and menstruation [1,2]. Consequently, the failure of "Jin" directly leads to edema or dehydration, frequent urination, hypoacusis, and osteoporosis, in addition to aging-related

\section{Journal of}

Integrative Medicine \& Therapy

Seiichiro Nishida ${ }^{1 *}$, Katsuharu Tsuchida ${ }^{2}$ and Hiroyasu Satoh ${ }^{3}$

${ }^{1}$ Koriyama Seiran Hospital, Yamatokoriyama, Nara 639-1136, Japan ${ }^{2}$ Rational Medicinal Science, Doshisha Women's College, Kyotanabe, Kyoto 610-0395, Japan

${ }^{3}$ Health Life Science, Shitennoji University, Habikino, Osaka 5838501, Japan

\section{*Address for Correspondence}

Seiichiro Nishida, Koriyama Seiran Hospital, Yamatokoriyama, Nara 6391136, Japan, Tel: +81-0743-56-8000; Fax: +81-0743-59-0022; E-mail: drmaro@m4.kcn.ne.jp

Submission: 04 March 2015

Accepted: 30 March 2015

Published: 03 April 2015

Reviewed \& Approved by: Dr. Harold H. Fain, Assistant Professor of Community Medicine, University of North Texas Health Science Center, USA

dysfunctions. Rokumigan can regulate "Jin" functions, resulting in rehabilitation of the body that is exhausted by water imbalance, chronic inflammatory disorders, and unusual heat. Rokumigan is traditionally believed to be effective for children who are suffering from unusual fever. Of late, however, it is considered useful for adults as well as children $[1,2]$.

On the other hand, both hachimijiogan and goshajinkigan have been traditionally prescribed for elderly individuals with the conditions such as dry skin, dry mouth, weak limbs, benign prostatic hypertrophy, and low back pain [1]. These conditions are primarily considered to be induced by decreased body temperature. Hachimijiogan and goshajinkigan can increase the body temperature and, as a result, ameliorate these conditions. Both drugs also resolve urinary abnormalities such as frequent urination or lower urinary tract symptoms [1,2]. Hachimijiogan relaxes acetylcholine (ACh)-induced contraction of isolated rat bladder strips [3], while goshajinkigan has been shown to be very effective for overactive bladder $(\mathrm{OAB})$ in male [4] and female patients [5], through regulation of bladder activity via the sympathetic and parasympathetic nervous systems [6] and suppression of C-fiber activation [7]. Therefore, the indications of both drugs are very similar, although goshajinkigan is relatively useful for elderly individuals suffering from low back pain and leg pain [1]. From our previous findings, increased effectiveness in elderly individuals is dependent on the number of crude drugs in a formulation [8].

The three above mentioned formulations have similar clinical indications, with all three regulating edema and water balance, possibly through an improvement in blood flow. Hachimijiogan increases the human retinal blood flow [9], while goshajinkigan increases the peripheral tissue blood flow in hind paws through nitric oxide (NO) production in diabetic rats [10]. These findings demonstrate that these formulations dilate blood vessels and increase blood flow. Although the usefulness has been reported in several 
Citation: Nishida S, Tsuchida K, Satoh H. Comparative Study of Vasodilatation Induced by Rokumigan, Hachimijiogan, and Goshajinkigan in the Rat Aorta. J Integrative Med Ther. 2015;2(1): 6.

ISSN: 2378-1343

studies, the in vitro vascular pharmacological actions remain unclear. Therefore, we compared the vasodilating effects and investigated the underlying mechanisms of the three Kampo formulations (rokumigan, hachimijiogan and goshajinkigan) using rat aorta strips.

\section{Materials and Methods}

All experiments were conducted according to the guidelines laid down by the Nara Medical University Animal Welfare Committee and the Doshisha Women's College Animal Welfare Committee, in compliance with tenets of the Declaration of Helsinki.

Male Wistar rats (10 to 15 weeks old) were anesthetized with ether and euthanized by exsanguination. The thoracic aorta was quickly removed and cut into ring strips measuring $3 \mathrm{~mm}$ in length. The experiments were conducted using previously described method $[8,11]$. The vascular strips were suspended between lower and upper triangular stainless steel stirrups in a jacketed organ chamber filled with $20 \mathrm{~mL}$ of modified Krebs- Henseleit solution. The lower stirrup was anchored and the upper stirrup was attached to a forcedisplacement transducer (Nihon Kohden TB-652T, Tokyo, Japan) to record the isometric force. All rings were stretched to generate a resting tension of $1.2 \mathrm{~g}$. The modified Krebs- Henseleit solution was composed of $118 \mathrm{NaCl}, 4.6 \mathrm{KCl}, 1.2 \mathrm{MgSO}_{4}, 1.2 \mathrm{KH}_{2} \mathrm{PO}_{4}, 11.1$ glucose, 27.2 $\mathrm{NaHCO}_{3}, 0.03 \mathrm{Na}_{2}$-ethylnediaminetetraacetic acid (EDTA), and $1.8 \mathrm{mM} \mathrm{CaCl}_{2}$. The chamber solution was maintained at $37{ }^{\circ} \mathrm{C}$ and oxygenated with $95 \% \mathrm{O}_{2}$ and $5 \% \mathrm{CO}_{2}$. After $40 \mathrm{~min}$ of resting, $5 \mu \mathrm{M}$ norepinephrine (NE; Daiichi Sankyo Co., Tokyo, Japan) was added to the tissue bath. After the contractile response became steady, the drugs were cumulatively added to the bath solution. The effects of the drugs were measured 10-20 min after the responses became steady. The relaxation responses were calculated as the percentage decreases relative to the maximal contraction induced by NE. The endothelium was carefully removed by rubbing the internal lumen with smooth and thin plastic sticks. The absence of a functional endothelium was confirmed by the lack of relaxation in response to acetylcholine (ACh, $1 \mu \mathrm{M})$.

Rokumigan (TJ-87), hachimijiogan (TJ-7), and goshajinkigan (TJ-107) are generally provided by Tsumura Co. (Tokyo, Japan) for clinical use in Japan. They are spray-dried powders extracted from boiling water with a mixture of the medical herbs. The drugs used in this study were supplied by Tsumura Co., and their components are summarized in Table 1. The Kampo formulations were dissolved in dimethyl sulfoxide (DMSO; Wako Chemical, Osaka, Japan). $\mathrm{N}^{\mathrm{G}}$-Nitro-L-arginine methyl ester (L-NAME), indomethacin, nicardipine and propranolol (all from Wako Chemical) were used for pretreatment. All values are represented as means \pm standard errors of the means. The differences in mean values were analyzed using analysis of variance (ANOVA) and Student's t-test. A p-value of < 0.05 was considered statistically significant.

\section{Results}

\section{Vasodilatation induced by rokumigan, hachimijiogan, and goshajinkigan}

Application of NE caused remarkable vasoconstriction of the resting aorta, while the formulations counteracted this vasodilatation by causing vasodilatation in a concentration-dependent manner (Table 2). Rokumigan induced less vasodilatation at $0.03 \mathrm{mg} / \mathrm{mL}$ $(0.51 \pm 0.25 \% ; \mathrm{n}=8)$ and significant vasodilatation at $>0.1 \mathrm{mg} / \mathrm{mL}(69.3$ $\pm 3.9 \%$ at $3.0 \mathrm{mg} / \mathrm{mL} ; \mathrm{n}=8 ; \mathrm{p}<0.01)$. Hachimijiogan also induced significant vasodilatation at $0.1-3.0 \mathrm{mg} / \mathrm{mL}(67.0 \pm 4.9 \%$ at $3 \mathrm{mg} / \mathrm{mL}$; $\mathrm{n}=8 ; \mathrm{p}<0.01)$. Similarly, goshajinkigan induced vasodilatation at $0.1-3.0 \mathrm{mg} / \mathrm{mL}(58.1 \pm 2.9 \%$ at $3 \mathrm{mg} / \mathrm{mL}: \mathrm{n}=8, \mathrm{p}<0.01)$. At $3.0 \mathrm{mg} /$ $\mathrm{mL}$, compared to the other two formulations, rokumigan induced the greatest vasodilatation $(\mathrm{n}=8 ; \mathrm{p}<0.05)$.

Table 1: The composition of the crude drugs contained in rokumigan, hachimijiogan and goshajinkigan.

\begin{tabular}{|c|c|c|c|}
\hline & rokumigan & hachimijiogan & goshajinkigan \\
\hline Rahmanniae Radix & $5.0 \mathrm{~g}$ & $6.0 \mathrm{~g}$ & $5.0 \mathrm{~g}$ \\
\hline Corni Fuructus & 3.0 & 3.0 & 3.0 \\
\hline Alismatis Rhizoma & 3.0 & 3.0 & 3.0 \\
\hline Poria cocos & 3.0 & 3.0 & 3.0 \\
\hline Moutan Cortex & 3.0 & 3.0 & 3.0 \\
\hline Processi Aconiti Tuber & 0.5 & 1.0 & \\
\hline Achyranthis Radix & 3.0 & & \\
\hline Plantaginis Semen & 3.0 & & \\
\hline
\end{tabular}

Rokumigan (TJ-87), hachimijiogan (TJ-7), and goshajinkigan (TJ-107) (Tsumura Co., Tokyo) were used.

Table 2: Comparison of the vasodilataion induced by rokumigan, hachimijiogan, and goshajinkigan.

\begin{tabular}{|c|c|c|c|c|c|}
\hline & $\mathbf{N}$ & $\mathbf{0 . 0 3}$ & $\mathbf{0 . 1}$ & $\mathbf{0 . 3}$ & $\mathbf{3 . 0} \mathbf{~ m g / m l}$ \\
\hline rokumigan & 8 & $0.5 \pm 0.25$ & $3.3 \pm 1.0^{*}$ & $12.7 \pm 2.1^{* *}$ & $28.7 \pm 3.4^{* *}$ \\
\hline hachimijiogan & 8 & $1.5 \pm 0.79$ & $3.5 \pm 1.3^{*}$ & $15.1 \pm 3.1^{* *}$ & $34.0 \pm 4.5^{* *}$ \\
\hline goshajinkigan & 8 & $1.4 \pm 0.99$ & $4.7 \pm 1.5^{*}$ & $11.5 \pm 2.3^{* *}$ & $24.7 \pm 2.5^{* *}$ \\
\hline
\end{tabular}

Values (\%) represent mean \pm S.E.M. ${ }^{*}$ and a): $p<0.05,{ }^{* *}$ : $p<0.01$. Symbols of ${ }^{*}$ and ${ }^{* *}$ mean a significance of comparison between the vasodilating effect at the each concentration and the maximal contraction induced by NE. Symbol of a means the significance of comparison between the values of rokumigan and goshajinkigan at the same concentration. 
Citation: Nishida S, Tsuchida K, Satoh H. Comparative Study of Vasodilatation Induced by Rokumigan, Hachimijiogan, and Goshajinkigan in the Rat Aorta. J Integrative Med Ther. 2015;2(1): 6.

Endothelium-dependent vasodilatation induced by rokumigan, hachimijiogan, and goshajinkigan

To investigate the endothelium dependency of vasodilatation, L-NAME $(100 \mu \mathrm{M})$, indomethacin $(10 \mu \mathrm{M})$, and endothelium removal were used as pretreatments. L-NAME, an NO synthesis inhibitor, attenuated 1.0 and $3.0 \mathrm{mg} / \mathrm{mL}$ rokumigan-induced vasodilatation from $28.7 \pm 3.4 \%$ to $18.3 \pm 2.7 \%(\mathrm{n}=8 ; \mathrm{p}<0.05)$ and from $69.7 \pm 3.0 \%$ to $46.4 \pm 3.5 \%(n=8 ; p<0.01)$, respectively. Indomethacin, an inhibitor of prostanoid production, showed no effect on vasodilatations at any concentration $[26.5 \pm 4.9 \%(\mathrm{n}=6)$ at $1.0 \mathrm{mg} / \mathrm{mL}$ and $68.9 \pm 5.2 \%(\mathrm{n}$ $=6$ ) at $3.0 \mathrm{mg} / \mathrm{mL}$ after indomethacin administrations]. On the other hand, endothelium removal significantly attenuated rokumiganinduced vasodilatation, which was $17.8 \pm 3.2 \%(\mathrm{n}=6: \mathrm{p}<0.05)$ at $1.0 \mathrm{mg} / \mathrm{mL}$ and $54.6 \pm 4.2 \%(\mathrm{n}=6 ; \mathrm{p}<0.05)$ at $3.0 \mathrm{mg} / \mathrm{mL}$ after endothelium removal (Figure 1A).

Hachimijiogan- and goshajinkigan-induced vasodilatation was also attenuated by pretreatment with both L-NAME and endothelium removal. Endothelium removal attenuated the vasodilatation induced by hachimijiogan at $0.3-3.0 \mathrm{mg} / \mathrm{mL}$, while L-NAME attenuated the vasodilatation induced by $1.0 \mathrm{mg} / \mathrm{mL}$ of hachimijiogan to $21.1 \pm$
$2.4 \%(\mathrm{n}=8 ; \mathrm{p}<0.05)$ and $3.0 \mathrm{mg} / \mathrm{mLof}$ hachimijiogan to $52.9 \pm 3.3 \%$ $(\mathrm{n}=8 ; \mathrm{p}<0.05$; Figure 1B). Goshajinkigan $(3.0 \mathrm{mg} / \mathrm{mL})$-induced vasodilatation decreased to $43.7 \pm 1.3 \%(\mathrm{n}=8 ; \mathrm{p}<0.01)$ in the presence of L-NAME and to $47.6 \pm 3.9 \%(n=8 ; p<0.01)$ under the condition of endothelium removal (Figure 1C). However, indomethacin showed no effects at any concentrations of both formulations. These results indicate that all three formulations induced endothelium-dependent vasodilatation, with no prostanoid involvement.

Endothelium-independent vasodilatation induced by rokumigan, hachimijiogan, and goshajinkigan

To examine the involvement of $\beta$-adrenoceptors and $\mathrm{Ca}^{2+}$ channels in the vasodilatation, propranolol $(3 \mu \mathrm{M})$ and nicardipine $(2 \mu \mathrm{M})$ were used for pretreatment. In the presence of propranolol $(3 \mu \mathrm{M})$, the vasodilatation induced by $3.0 \mathrm{mg} / \mathrm{mL}$ of rokumigan was attenuated to $55.2 \pm 4.5 \%(\mathrm{n}=6 ; \mathrm{p}<0.05$; Figure $2 \mathrm{~A})$. Furthermore, nicardipine remarkably attenuated the vasodilatation induced by 0.3 , 1.0 , and $3.0 \mathrm{mg} / \mathrm{mL}$ of rokumigan to $3.9 \pm 2.4 \%(\mathrm{n}=6 ; \mathrm{p}<0.01), 12.4$ $\pm 4.9 \%(\mathrm{n}=6 ; \mathrm{p}<0.01)$, and $51.2 \pm 7.4 \%(\mathrm{n}=6 ; \mathrm{p}<0.05)$, respectively (Figure $2 \mathrm{~A}$ ). In $\mathrm{Ca}^{2+}$-free solutions, the vasodilatation induced by rokumigan is attenuated at $0.3 \sim 3 \mathrm{mg} / \mathrm{ml}$ significantly. In $\mathrm{Ca}^{2+}$-free
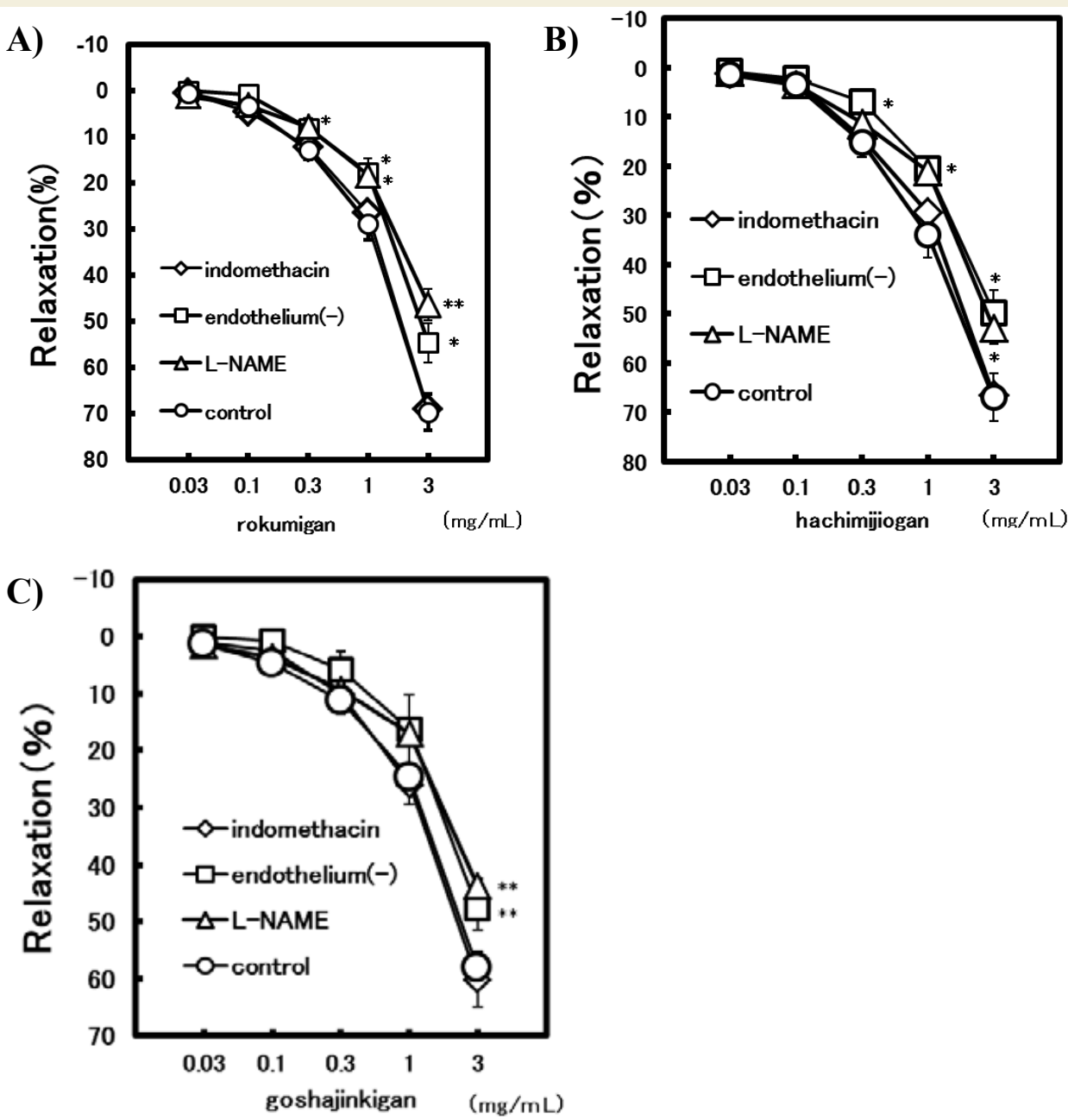

Figure 1: Endothelium-dependent vasodilatation induced by rokumigan, hachimijiogan, and goshajinkigan. 

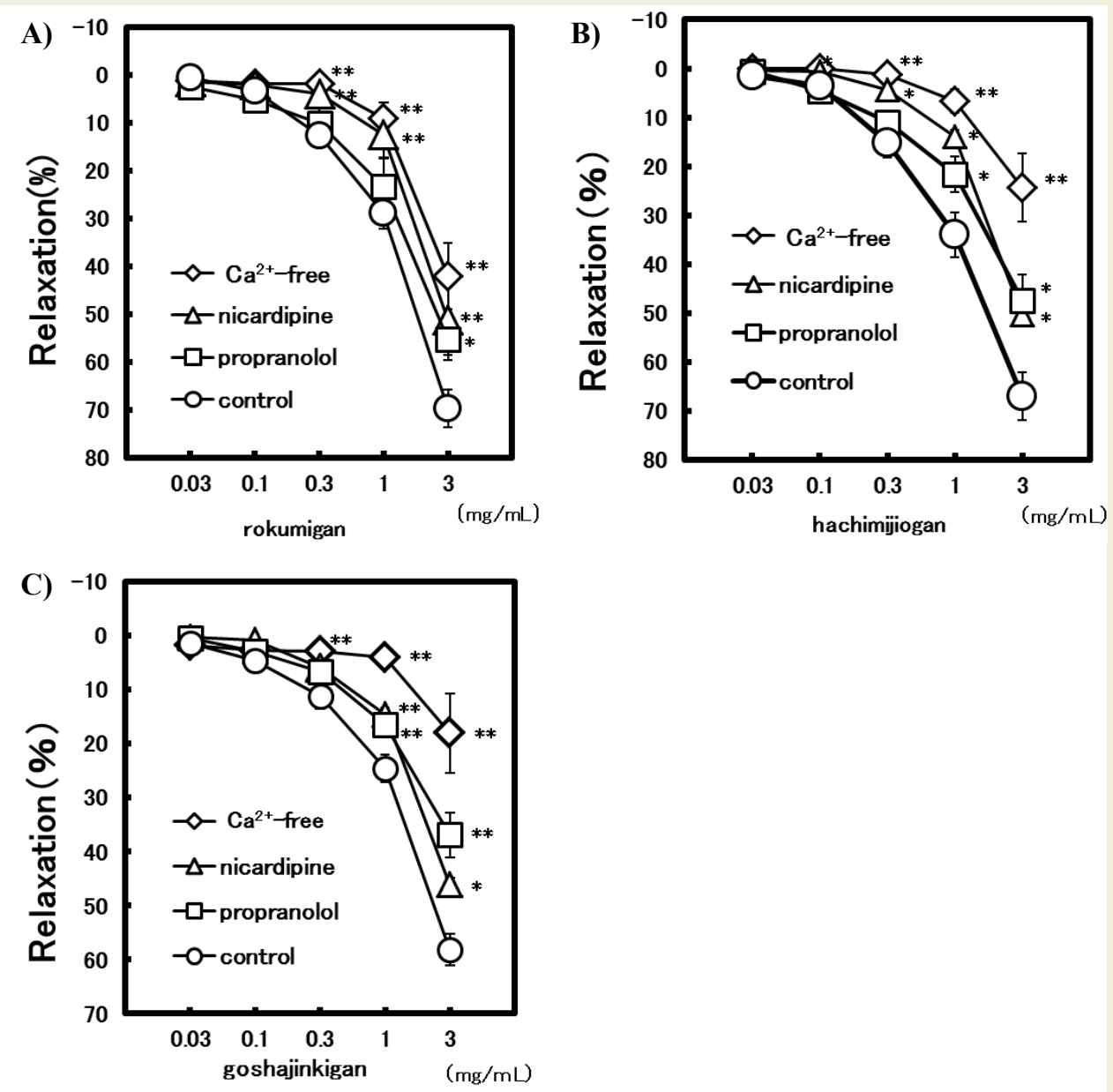

Figure 2: Modulation of endothelium-independent vasodilatation induced by rokumigan, hachimijogan and goshajinkigan.

solution, the vasodilatation-induced by $0.3,1.0$, and $3.0 \mathrm{mg} / \mathrm{mL}$ of rokumigan was significantly attenuated to $1.8 \pm 1.3 \%(\mathrm{n}=6$; $\mathrm{p}<$ $0.01), 8.9 \pm 3.2 \%(\mathrm{n}=6 ; \mathrm{p}<0.01)$, and $41.9 \pm 6.9 \%(\mathrm{n}=6 ; \mathrm{p}<0.01)$, respectively (Figure $2 \mathrm{~A}$ ). This result also indicates the involvement of $\mathrm{Ca}^{2+}$ channels in rokumigan-induced vasodilatation. Furthermore, propranolol $(3 \mu \mathrm{M})$ significantly attenuated hachimijiogan- and goshajinkigan-induced vasodilatation at 1.0 and $3.0 \mathrm{mg} / \mathrm{mL}$ (Figures $2 \mathrm{~B}$ and $2 \mathrm{C})$, while nicardipine $(2 \mu \mathrm{M})$ showed the same effects on vasodilatation induced by the two formulations at 0.3 to $3.0 \mathrm{mg} / \mathrm{mL}$ (Figures $2 \mathrm{~B}$ and $2 \mathrm{C}$ ). In $\mathrm{Ca}^{2+}$-free solutions as well, the vasodilatation induced by $0.3-3.0 \mathrm{mg} / \mathrm{mL}$ of hachimijiogan and goshajinkigan was markedly attenuated (Figures $2 \mathrm{~B}$ and $2 \mathrm{C}$ ). These results demonstrate that rokumigan, hachimijiogan, and goshajinkigan relax the vascular smooth muscle cells through $\beta$-adrenoceptor stimulation and $\mathrm{Ca}^{2+}$ channel inhibition.

\section{Discussion}

The present experiments showed that rokumigan, hachimijiogan, and goshajinkigan dilated the rat aorta constricted by $\mathrm{NE}$ in a concentration-dependent manner; that the vasodilatations induced by all three formulations were similar, although rokumigan at $3.0 \mathrm{mg} /$ $\mathrm{mL}$ showed the greatest vasodilatation; that the three formulations induced endothelium-dependent vasodilatation that was primarily mediated by NO; that the vasodilatation was not dependent on prostaglandin; and that the Kampo formulations induced vascular smooth muscle relaxation through $\mathrm{Ca}^{2+}$ channel inhibition and $\beta$-adrenoceptor stimulation.

The clinical indication of rokumigan, hachimijiogan and goshajinkigan are similar, with their effects on edema and decreased body temperature partially attributed to their vasodilating effects. Although there are some differences among the components of the three kampo formulations, the vasodilatations profiles were almost similar in this study. As a whole, vasodilatation results from complex interactions among the crude drugs included in each formulation [8]. Rokumigan basically comprises six crude drugs. In hachimijiogan, two more crude drugs, Cinnamomi Cortex and Processi Aconiti Tuber, are added to the six basic crude drugs of rokumigan, while two additional crude drugs are added to goshajinkigan, which comprises a total of 10 crude drugs. Our previous findings indicated that increased effectiveness in elderly rats with an increase in the number of crude drugs in a formulation [8]. However, in young rats (10-15 weeks old), the strength of vasodilatation induced by rokumigan, hachimigan, and goshajinkigan was almost independent of the number of crude drugs in each formulation. 
Citation: Nishida S, Tsuchida K, Satoh H. Comparative Study of Vasodilatation Induced by Rokumigan, Hachimijiogan, and Goshajinkigan in the Rat Aorta. J Integrative Med Ther. 2015;2(1): 6.

ISSN: $2378-1343$

Rokumigan, hachimijiogan, and goshajinkigan may exhibit various pharmacological potencies. These Kampo formulations produce NO from the vascular endothelium. The antinocieptive effects of goshajinkigan in diabetic mice may have partially resulted from peripheral production of NO [13].

The vasodilating effects described above lead to multiple pharmacological actions, including improved peripheral and cerebral blood flow and cerebral functions and decreased edema. Hachimijiogan and goshajinkigan warm the extremities and the body $[1,2]$. Moreover, rokumigan [14] and hachimijiogan [15] have shown usefulness for memory enhancement, improvement of precognitive abilities, prevention of neuronal degeneration [16], and protection from ischemia/reperfusion-induced renal injury [17].

Most recently, the effects of hachimijiogan and goshajinkigan on diabetes were shown $[18,19]$. Hachimijiogan improved insulin secretion and glucose tolerance in rats with spontaneous type-2 diabetes [20], while goshajinkigan improved insulin resistance via the NO pathway in rats with diabetes $[19,21]$. Furthermore, it was recently found that both formulations are beneficial for glycemic regulation in rats with diabetes [22] and for diabetic nephropathy [23].

With regard to the ingredients, the NO dependency of Corni Fuructus [24] and the $\mathrm{Ca}^{2+}$ channel inhibition effects of Alismatis Rhizoma [25] have been demonstrated, consistent with the findings of the present study. Cinnamomi Cortex and Processi Aconiti Tuber also possess potent vasodilating effect. Cinnamaldehyde, one of the ingredients in Cinnamomi cortex, exerts vasodilating effects via endothelium-derived relaxation factor (NO) and by blocking $\mathrm{Ca}^{2+}$ channels, although indomethacin does not affect cinnamaldehydeinduced vasodilatation [26]. Mesaconitine, the main ingredient of Aconiti tuber, increases the intracellular $\mathrm{Ca}^{2+}$ concentration $\left(\left[\mathrm{Ca}^{2+}\right]_{\mathrm{i}}\right)$ in endothelial cells. The increase of $\left[\mathrm{Ca}^{2+}\right]_{\mathrm{i}}$ stimulates NO production and induces vasodilatation [27]. These findings strongly support the present results. In the rat mesenteric artery, mesaconitine induces vasodilatation mediated by the endothelium-derived hyperpolarization factor (EDHF) [28]. The vasodilating mechanisms of the previously reported ingredients are summarized in Table 3.

The clinically effective concentrations of the constituents and ingredients (phytochemicals) in the three formulations described

Table 3: Ingredients of the crude drugs and their vasodliating mechanisms.

\begin{tabular}{|c|c|c|}
\hline & $\begin{array}{c}\text { Main ingredient } \\
\text { for vasodilataion }\end{array}$ & $\begin{array}{l}\text { Mechanisms for } \\
\text { vasodilatation }\end{array}$ \\
\hline Rehmanniae Radix & & unknown \\
\hline Corni Fuructus & Cornuside & NO (EDRF) \\
\hline Dioscorea Rhizoma & & unknown \\
\hline Alismatis Rhizoma & Alismol & $\begin{array}{l}\mathrm{Ca}^{2+} \text { channel } \\
\text { inhibition }\end{array}$ \\
\hline Poria cocos & & unknown \\
\hline Moutan Cortex & & unknown \\
\hline Cinnamomi Cortex channel. & Cinnamaldehyde & $\begin{array}{l}\text { NO-dependent/ } \\
\text { blocking } \mathrm{Ca}^{2+}\end{array}$ \\
\hline Processi Aconiti Tuber & Mesaconitine & NO/EDHF \\
\hline Achyranthis Radix & & unknown \\
\hline Plantaginis Semen & & unknown \\
\hline
\end{tabular}

here remain unclear. In this study, we qualitatively evaluated the vasodilating effects of these formulations, and there is no report on the serum concentrations of these Kampo formulations after oral intake, presumably because so many ingredients exist as crude drug and Kampo formulations. The concentrations of formulations used in this study would be higher than the clinical concentrations used for oral administration. In general, the concentrations in vitro experiments are relatively higher, because the pharmacological responses occur and are observed as acute responses within the limited time.

In conclusion, rokumigan, hachimijiogan and goshajinkigan show significant vasodilating effects due to NO released by the endothelium and due to inhibition of L-type $\mathrm{Ca}^{2+}$ channels and stimulation of $\beta$-adrenoceptors on vascular smooth muscles. These Kampo formulations show multiple pharmacological actions, with their vasodilating effects contributing to some of these actions, such as regulation of edema, peripheral blood flow, and cerebral function. For a more comprehensive understanding of the clinical contributions of these Kampo formulations, further in vivo experiments are required. Moreover, the effects of the ingredients in these Kampo formulations should be elucidated.

\section{References}

1. Y Sato (2005) Introduction of Kampo: Japanese traditional medicine. Elsevier, Tokyo, Japan.

2. Y Shibata, Jean Wu (1997) Kampo treatment for climatic disorders: a hand book for practioners. Paradigm Publications, Massachusetts, USA.

3. Ito Y, Seki M, Nishioka Y, Kumura M, Yasuda A, et al. (2009) Pharmacological effects of Hachi-mi-jio-gan extract (Harncare) on the contractile response and on pharmacologically relevant receptors in the rat bladder. Yakugaku Zasshi 129: 957-964.

4. Ogushi T, Takahashi S (2007) Effect of chinese herbal medicine on overactive bladder. Hinyokika Kiyo 53: 857-862.

5. Kajiwara M, Mutaguchi K (2008) Clinical efficacy and tolerability of goshajinki-gan, Japanese traditional herbal medicine, in females with overactive bladder. Hinyokika Kiyo 54: 95-99.

6. Nishijima S, Sugaya K, Miyazato M, Ogawa Y (2007) Effect of Gosha-jinkigan, a blended herbal medicine, on bladder activity in rats. J Urol 177: 762765.

7. Imamura T, Ishizuka O, Aizawa N, Zhong C, Ogawa T, et al. (2008) Goshajinki-gan reduces transmitter proteins and sensory receptors associated with $\mathrm{C}$ fiber activation induced by acetic acid in rat urinary bladder. Neurourol Urodyn 27: 832-837.

8. Satoh H (2013) Pharmacological characteristics of Kampo medicine as a mixture of constituents and ingredients. J Integr Med 11: 11-16.

9. Isobe H, Yamamoto K, Cyong JC (2003) Effects of hachimi-jio-gan (ba-weidi-huang-wan) on blood flow in the human central retinal artery. Am $\mathrm{J}$ Chin Med 31: 425-435

10. Suzuki Y, Goto K, Ishige A, Komatsu Y, Kamei J (1998) Effects of goshajinki-gan, a kampo medicine, on peripheral tissue blood flow in streptozocininduced diabetic rats. Methods Find Exp Clin Pharmacol 20: 321-328.

11. Nishida S, Satoh $H(2006)$ In vitro pharmacological actions of sinomenine on the smooth muscle and the endothelial cell activity in rat aorta. Life Sci 79 : 1203-1206.

12. Nishida S, Satoh H (2007) Vascular pharmacology of mokuboito (mu-fang-yitang) and its constituents on the smooth muscle and the endothelium in rat aorta. Evid Based Complement Alternat Med 4: 335-341.

13. Suzuki Y, Goto K, Ishige A, Komatsu Y, Kamei J (1999) Antinocieptive mechanism of gosya-jinki-gan: role of nitric oxide in the periphery. Jpn J Pharmacol 79: 387-391. 
Citation: Nishida S, Tsuchida K, Satoh H. Comparative Study of Vasodilatation Induced by Rokumigan, Hachimijiogan, and Goshajinkigan in the Rat Aorta. J Integrative Med Ther. 2015;2(1): 6.

14. Park E, Kang M, Oh JW, Park C, Kim C, et al. (2005) Yukmijihwang-tang derivatives enhance cognitive processing in normal young adults: a doubleblinded, placebo-controlled trial. Am J Chin Med 33: 107-115.

15. Iwasaki K, Kobayashi S, Chimura Y, Taguchi M, Inoue K, et al. (2004) A randomized, double-blind, placebo-controlled clinical trial of the Chinese herbal medicine "ba wei di huang wan" in the treatment of dementia. J Am Geriatr Soc 52: 1518-1521.

16. Rho S, Kang M, Choi B, Sim D, Lee J, et al. (2005) Effects of Yukmijihwangtang derivatives (YMJd), a memory enhancing herbal extract, on the geneexpression profile in the rat hippocampus. Biol Pharml Bull 28: 87-93.

17. Kang DG, Sohn EJ, Moon MK, Mum YJ, Woo MK, et al. (2006) Yukmijihwang tang ameliorates ischemia/reperfusion-induced renal injury in rats. $J$ Ethnopharmacol 104: 47-53.

18. Wu YC, Hsu JH, Liu IM, Liou SS, Su HC, et al. (2002) Increase of insulin sensitivity in diabetic rats received Die-Huang-Wan, a herbal mixture used in Chinese traditional medicine. Acta Pharmacol Sin 23: 1181-1187.

19. Hu X, Sato J, Bajotto G, Khookhor O, Ohsawa I, et al. (2010) Goshajinkigan (Chinese herbal medicine Niu-Che-Sen-Qi-Wan) improves insulin resistance in diabetic rats via the nitric oxide pathway. Nagoya J Med Sci 72: 35-42.

20. Hirotani Y, Ikeda K, Myoutoku M (2010) Effects of the herbal medicine Hachimi-jio-gan (Ba-Wei-Di-Huang-Wan) on insulin secretion and glucose tolerance in type 2 diabetic Goto-Kakizaki rats. Drug Discov Ther 4: 129-134.

21. Hu X, Sato J, Bajotto G, Khookhor O, Ohsawa I, et al. (2010) Goshajinkigan (Chinese herbal medicine niu-che-sen-qi-wan) improves insulin resistance in diabetic rats via the nitric oxide pathway. Nagoya J Medl Sci 72: 35-42.
22. Hirotani Y, Ikeda T, Yamamoto K, Kurokawa N (2007) Effects of Hachimijio-gan (Ba-Wei-Di-Huang-Wan) on hyperglycemia in streptozotocin-induced diabetic rats. Biol Pharml Bull 30: 1015-1020.

23. Watanabe K, Shimada A, Miyaki K, Hirakata A, Matsuoka K, et al. (2014) Long-term effects of goshajinkigan in prevention of diabetic complications: a randomized open-labeled clinical trial. Evid Based Complement Alter Med 2014: 128726.

24. Kang DG, Choi DH, Lee JK, Lee YJ, Moon MK, et al. (2007) Endothelial NO/ cGMP-dependent vascular relaxation of cornuside isolated from the fruit of Cornus officials. Planta Med 73: 1436-1440.

25. Matsuda H, Kobayashi G, Yamahara J, Fujimura H, Kurahashi K, et al. (1987) Effects of alismol isolated from Alismatis Rhizoma on calcium-induced contraction in the rabbit thoracic aorta. Life Sci 41: 1845-1852.

26. Yanaga A, Goto H, Nakagawa T, Hikiami H, Shibahara N, et al. (2006) Cinnamaldehyde induces endothelium-dependent and -independent vasorelaxant action on isolated rat aorta. Biol Pharml Bull 29: 2415-2418.

27. Mitamura M, Horie S, Sakaguchi M, Someya A, Tsuchiya S, et al. (2002) Mesaconitine-induced relaxation in rat aorta: involvement of $\mathrm{Ca}^{2+}$ influx and nitric-oxide synthase in the endothelium. Eur J Pharmacol 436: 217-225.

28. Mitamura M, Boussery K, Horie S, Murayama T, Van de Voorde J, et al. (2002) Vasorelaxing effect of mesaconitine, an alkaloid from Aconitum japonicum, on rat small gastric artery: possible involvement of endotheliumderived hyperpolarizing factor. Jpn J Pharmacol 89: 380-387.

\section{Acknowledgements}

The authors wish to thank tsumura Co. for providing kampo formulations, namely rokumigan (TJ-87), hachimijiogan (TJ-7), and goshajinkigan (TJ-107). 